\title{
Spectral Clustering of Images in LUV Color Space by Spatial-Color Pixel Classification
}

\author{
C.P. Blesslin Elizabeth \\ P.G Scholar, Applied Electronics \\ Anna University, Tirunelveli, India
}

\author{
K. Usha Kingsly Devi \\ Assistant professor, ECE Department \\ Anna University, Tirunelveli, India
}

\begin{abstract}
This work is based on color image segmentation by spatial-color pixel classification in Luv color space. Classes of pixels are difficult to be identified when the color distributions of the different objects highly overlap in the color space and when the color points give rise to non-convex clusters. It is proposed to apply spectral classification to regroup the pixels which represent the same regions, into classes. Spectral clustering achieves a spectral decomposition of a similarity matrix in order to construct an eigen-space in which the clusters are expected to be well separated. The similarity matrix used in this paper is derived from a spatial-color compactness function. This function takes into account both the distribution of colors in the color space and the spatial location of colors in the image plane. Spectral clustering that uses FCM performs better in Luv color space when compared with other Spectral clustering algorithms..
\end{abstract}

\section{Keywords:}

Spectral Clustering, Non-convex clusters, Eigen-Space

\section{INTRODUCTION}

The segmentation of a color image consists in partitioning it into separate regions, in order to extract the various objects of the represented scene. Clustering is one of the most widely used techniques for exploratory data analysis, with applications ranging from statistics, computer science, biology to social sciences or psychology. In virtually every scientific field dealing with empirical data, people attempt to get a first impression on their data by trying to identify groups of "similar behaviour" in their data. Spectral clustering is very simple to implement and can be solved efficiently by standard linear algebra methods.Spectral clustering is used to construct the pixel classes. The regions of the segmented image are composed of connected pixels which are assigned to the same classes. The spectral clustering is based on a spectral decomposition of a matrix of similarities between the data.

Classes of pixels are difficult to be identified in previous methods (Shi's and Ng's) when the color distributions of the different objects highly overlap in the color space and when the color points give rise to non-convex clusters. The objective of this paper is to segment the color image by spectral clustering using spatial-color compactness function. This involves computation of the similarity matrix between each pair of colors by considering their spatial-color compactness degrees. This is an extension of Shi's and Ng's methods which helps in identifying non-convex clusters by using distribution of colors in the color space and the spatial location of colors in the image plane.

\section{LITERATURE REVIEW}

Chen T.Q. et al (2002) proposed an innovative approach of color image segmentation [3]. It describes a color image segmentation system that performs color clustering in a color space and then color region segmentation in the image domain. This color image segmentation system is quite successful in finding structures of color regions, and is flexible in controlling color resolution, region size and color distances. Deng Y. et al (2001) proposed the unsupervised segmentation of color-texture regions in images and video [6]. This method, referred to as JSEG, consists of two independent steps: color quantization and spatial segmentation. Veronica S. Moertini (2002) proposed data clustering algorithms [13]. Clustering is one of the primary tasks of data mining. It introduces the most representative algorithms used in off-line mode that apply crisp or fuzzy approach. The algorithms are KMeans, Fuzzy C-Means (FCM), mountain, subtractive and Partition Simplification Fuzzy C-Means clustering. Comaniciu D. et al (2002) proposed mean shift, a robust approach towards feature space analysis [5]. This method is used for the analysis of a complex multimodal feature space and to delineate arbitrary shaped clusters in it.

Fischer A. Y. N. et al (2002) proposed analysis and algorithm on spectral clustering [7]. Using tools from matrix perturbation theory, the algorithm is analyzed and gives conditions under which it can be expected to do well. Shi J. et al (2000) proposed normalized cuts and image segmentation [11]. This method illustrates the novel approach for solving the perceptual grouping problem in vision.

Chang H. et al (2008) developed robust path-based spectral clustering [2]. Clustering has been among the most active research topics in machine learning and pattern recognition. The idea of M-estimation in robust statistics is to achieve more accurate similarity measure in the presence of outliers. This method is significantly more robust than spectral clustering and path-based clustering.

Macaire L. et al (2006) proposed connectedness and color homogeneity properties of Color image segmentation[10]. This method is a new color image segmentation scheme based on unsupervised pixel classification that works even when there is not a one-to-one correspondence between the clusters of color points in the color space and the regions in the image.

Botte-Lecocq C. et al (2007) proposed color image segmentation by compacigram analysis [14]. This method is based on color image segmentation by pixel classification. The compacigram takes into account both the distribution of colors in the color space and the spatial location of colors in the image plane. 


\section{PROPOSED WORK}

The RGB image is taken as input image. First step is to perform conversion of RGB to Luv color space. Then, Spatial-color compactness function is calculated for Luv image. Next, the spatial color compactness function is applied to spectral clustering. Finally image is segmented into regions. Figure 1 shows the flow chart for the proposed method.

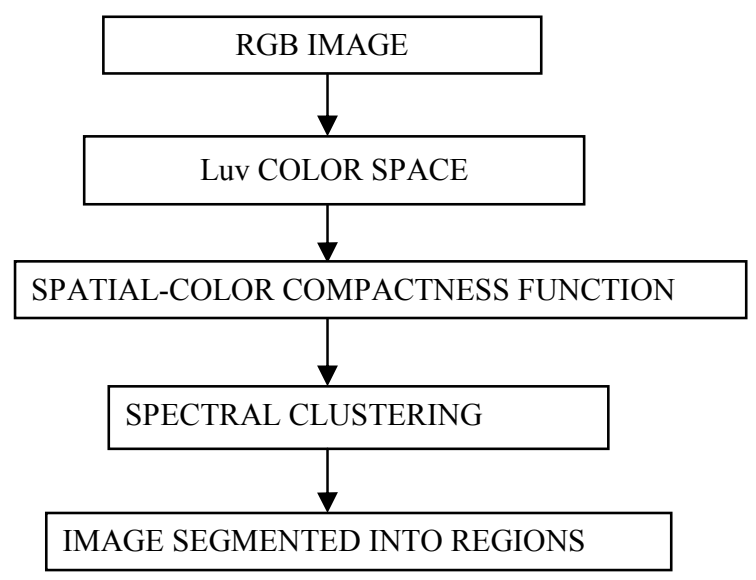

Figure 1.Flow Chart

\subsection{Conversion of RGB to Luv}

Given an RGB color whose components are in the nominal range $[0.0,1.0]$ and whose gamma is $\gamma$.

$$
\left[\begin{array}{l}
\mathrm{X} \\
\mathrm{Y} \\
\mathrm{Z}
\end{array}\right]=[\mathrm{M}]\left[\begin{array}{l}
\mathrm{r} \\
\mathrm{g} \\
\mathrm{b}
\end{array}\right]
$$

where, if the RGB system is not sRGB:

$$
\begin{aligned}
& \mathrm{r}=\mathrm{R}^{\gamma} \\
& \mathrm{g}=\mathrm{G}^{\gamma} \\
& \mathrm{b}=\mathrm{B}^{\gamma}
\end{aligned}
$$

and if it is sRGB:

$$
\begin{aligned}
& \mathrm{r}= \begin{cases}\mathrm{R} / 12.92 & \mathrm{R} \leq 0.04045 \\
((\mathrm{R}+0.055) / 1.0555)^{2.4} & \mathrm{R}>0.04045\end{cases} \\
& \mathrm{g}= \begin{cases}\mathrm{G} / 12.92 & \mathrm{G} \leq 0.04045 \\
((\mathrm{G}+0.055) / 1.0555)^{2.4} & \mathrm{G}>0.04045\end{cases} \\
& \mathrm{b}= \begin{cases}\mathrm{B} / 12.92 & \mathrm{~B} \leq 0.04045 \\
((\mathrm{~B}+0.055) / 1.0555)^{2.4} & \mathrm{~B}>0.04045\end{cases}
\end{aligned}
$$

This Conversion requires a reference white $\mathrm{X}_{\mathrm{r}}, \mathrm{Y}_{\mathrm{r}}, \mathrm{Z}_{\mathrm{r}}$

$$
L= \begin{cases}116 \sqrt[3]{y_{r}}-16 & y_{r}>\varepsilon \\ \kappa y_{r} & y_{r} \leq \varepsilon\end{cases}
$$

$$
\begin{aligned}
& \mathrm{u}=13 \mathrm{~L}\left(\mathrm{u}^{\prime}-\mathrm{u}_{\mathrm{r}}^{\prime}\right) \\
& \mathrm{v}=13 \mathrm{~L}\left(\mathrm{v}^{\prime}-\mathrm{v}_{\mathrm{r}}^{\prime}\right)
\end{aligned}
$$

Where,

$$
\begin{aligned}
& u^{\prime}=\frac{4 X}{X+15 Y+3 Z} \\
& v^{\prime}=\frac{4 Y}{X+15 Y+3 Z} \\
& u_{r}^{\prime}=\frac{4 X_{r}}{X_{r}+15 Y_{r}+3 Z_{r}} \\
& v_{r}^{\prime}=\frac{4 Y_{r}}{X_{r}+15 Y_{r}+3 Z_{r}}
\end{aligned}
$$

$$
\begin{aligned}
& \varepsilon=\left\{\begin{array}{cc}
0.008856 & \text { Actual CIE S tan dard } \\
216 / 24389 & \text { Intent of CIE S tan dard }
\end{array}\right. \\
& \kappa=\left\{\begin{array}{cl}
903.3 & \text { Actual CIE S tan dard } \\
24389 / 27 & \text { Intent of CIE S tan dard }
\end{array}\right.
\end{aligned}
$$

\subsection{Spatial-Color Compactness Function}

Let $\underline{D}(\mathrm{C}, 1)$ be a cube, centered at $\mathrm{C}=\left[\mathrm{C}^{\mathrm{R}}, \mathrm{C}^{\mathrm{G}}, \mathrm{C}^{\mathrm{B}}\right]^{\mathrm{T}}$ in the color space, whose edges of length 1 (an odd integer) are parallel to the $\mathrm{C}$ axes. The cube $\underline{\mathrm{D}}(\mathrm{C}, 1)$ therefore includes all the color points $\mathrm{c}=\left[\mathrm{c}^{\mathrm{R}}, \mathrm{c}^{\mathrm{G}}, \mathrm{c}^{\mathrm{B}}\right]^{\mathrm{T}}$ such that $\mathrm{C}^{\mathrm{i}}-\frac{1-1}{2} \leq \mathrm{c}^{\mathrm{i}} \leq \mathrm{C}^{\mathrm{i}}+\frac{1-1}{2}, \mathrm{i}=\mathrm{R}, \mathrm{G}, \mathrm{B}$. The image subset formed by all the pixels whose colors are included in the color domain $\underline{\mathrm{D}}(\mathrm{C}, 1)$ is hereafter referred to as $\mathrm{S}(\mathrm{C}, 1)$.

The connectedness degree $\mathrm{CD}(\mathrm{C}, 1)$ is defined as the average number of neighbors of each pixel of $\mathrm{S}(\mathrm{C}, 1)$ which also belong to $\mathrm{S}(\mathrm{C}, 1)$.A value of $\mathrm{CD}(\mathrm{C}, 1)$ close to 0 indicates that the pixels of $\mathrm{S}(\mathrm{C}, 1)$ are scattered in the image plane. On the opposite, a high value of the connectedness degree, close to 1 , means that the pixels belonging to the considered subset are highly connected to each other in the image.

The homogeneity degree $\operatorname{HD}(\mathrm{C}, 1)$ is defined as the ratio of the average local dispersion of colors in the neighborhood of each pixel in $\mathrm{S}(\mathrm{C}, 1)$, to the global color dispersion of the pixels belonging to $\mathrm{S}(\mathrm{C}, \mathrm{l})$. The above local dispersion at each pixel in 
$\mathrm{S}(\mathrm{C}, 1)$ only takes into account its neighbors that also belong to $\mathrm{S}(\mathrm{C}, 1)$.

When the color points corresponding to the pixels in the $\mathrm{S}(\mathrm{C}, 1)$ subset give rise to a compact cluster in the color space, the homogeneity degree is close to 1 . On the opposite, when those color points give rise to several distinct clusters, the homogeneity degree is low and close to 0 . The spatial-color compactness degree (SCCD) of a given color $\mathrm{C}$ as the product of its connectedness and homogeneity degrees:

$$
\operatorname{SCCD}(C, l)=C D(C, l) \times H D(C, l) .
$$

A high value of $\operatorname{SCCD}(C, 1)$ indicates that the pixels in the $\mathrm{S}(\mathrm{C}, 1)$ subset are highly connected in the image $(\mathrm{CD}(\mathbf{C}, \mathbf{l})$ close to 1) and that the color points corresponding to these pixels are concentrated in the color space $(\operatorname{HD}(\mathbf{C}, 1)$ close to 1$)$. Conversely, a low value of $\operatorname{SCCD}(\mathbf{C}, 1)$ means that the pixels in the $\operatorname{S}(\mathbf{C}, 1)$ subset are scattered in the image $(\operatorname{CD}(\mathbf{C}, 1)$ close to 0$)$ and/or that the color points of these pixels do not form a separate compact cluster in the color space $(\operatorname{HD}(\mathbf{C}, 1)$ close to 0$)$. In order to construct the classes, it is proposed to compute the similarity between each pairwise of colors by considering their spatial-color compactness degrees. For this purpose, consider each pair of colors $\mathrm{C}_{1}, \mathrm{C}_{2}$ and measure the similarity $\Phi\left(\mathrm{C}_{1}, \mathrm{C}_{2}\right)$ as:

$$
\Phi\left(C_{1}, C_{2}\right)=\min _{C \in \underline{D}\left(C_{1}, C_{2}\right)} \operatorname{SCCD}(C, l)
$$

Where, $\underline{\mathrm{D}}\left(\mathrm{C}_{1}, \mathrm{C}_{2}\right)$ is the parallelepiped color domain bounded by $\mathrm{C}_{1}$ and $\mathrm{C}_{2}$.

\subsection{Spectral Clustering}

Spectral Clustering algorithms are composed of three main successive steps. First, a similarity (or an affinity) matrix is computed between each pairwise of data points. The second step consists in extracting the $\mathrm{k}$ main eigenvectors of the similarity matrix, where $\mathrm{k}$ denotes the desired number of clusters. The clusters formed by data points projected in the eigenspace spanned by these $\mathrm{k}$ eigenvectors are expected to be convex. Therefore, a simple k-means achieved in the eigenspace succeeds in identifying the $\mathrm{k}$ expected clusters in the third step. Outside this ability to recognize non-convex clusters, it is interesting to note that the transformation of the initial set of points (second step) does not involve optimization techniques that may fail in case of local minima: one iteration is enough. In the next step as well, kmeans should not require a large number of iterations: the color points projected into the eigenspace should give rise to well separated compact clusters that should consequently be easily identified.

Given a set of $n$ points $C=\left\{\mathrm{C}_{1}, \mathrm{C}_{2}, \ldots, \mathrm{C}_{\mathrm{n}}\right\}$ in $\mathrm{R}^{3}$ to be classified into $\mathrm{k}$ classes:

Step1: To construct the similarity matrix $A \in R^{n \times n}$ defined by $A_{i j}$, a value of $A_{i j}$ close to 0 when the points $C_{i}$ and $C_{j}$ are different, whereas a value close to 1 when the colors are similar and merit to be regrouped into the same class.

Step2: Calculate the diagonal matrix $D \in R^{n \times n}$ by $D_{i i}=A_{i m}$, and define the normalized similarity matrix $\mathrm{L}=\mathrm{D}^{-1 / 2} \mathrm{AD}^{-1 / 2}$ Step3: Extract the $\mathrm{k}$ principal eigenvectors $\left\{\mathrm{X}_{1}, \ldots . ., \mathrm{X}_{\mathrm{k}}\right\}$ of $\mathrm{L}$, and form the matrix $\mathrm{X}$ containing the eigen vectors as columns.

Step4: Compute the matrix $\mathrm{Y}=\mathrm{D}^{-1 / 2} \mathrm{X}$ which is considered as a normalization of $\mathrm{X}$.

Step5: By considering that each point $\mathrm{C}_{\mathrm{i}} \mathrm{C} \mathrm{C}$ is transformed into $\mathrm{C}_{\mathrm{i}}^{\prime} \in \mathrm{R}^{\mathrm{k}}$ whose coordinates are: $\left\{\mathrm{Y}_{\mathrm{im}}, \mathrm{m} \in\{1, \ldots . ., \mathrm{k}\}\right\}$, apply a k-means clustering or FCM on this transformed set of points $\mathrm{C}^{\prime}$.

Step6: Finally assign the original point $C_{i}$ to the class at which its corresponding transformed point $\mathrm{C}_{\mathrm{i}}^{\prime}$ is assigned.

\section{EXPERIMENTAL RESULTS}

Figure 2 shows the input image of size 120x80. Results are produced by performing segmentation by four algorithms. In first algorithm (A1), above RGB image is taken as the input. Red and green components of the RGB image are taken separately. SCCD is calculated by the product of connectedness and homogeneity degree. SCCD is calculated for both the components. Similarity degree is calculated by taking the minimum of spatial-color compactness degrees of red and green components. From the similarity matrix, Eigen values and Eigen vectors are calculated. Only k principal Eigen values are considered. Normalisation of Eigen vectors takes place. Normalised matrix is applied to kmeans clustering. By means of $\mathrm{k}$-means clustering, image is segmented into regions. Finally the clusters are labeled. Figure 3 shows the output image of A1.

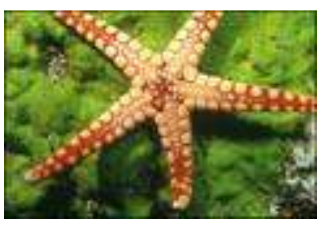

Figure 2.

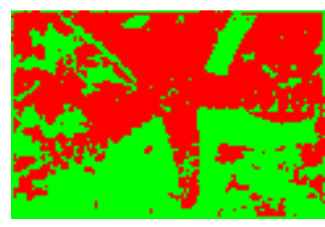

Figure 3.
In the second Algorithm (A2), the same procedure of (A1) is repeated with FCM instead of k-means clustering. Figure 4 shows the output image of A2.

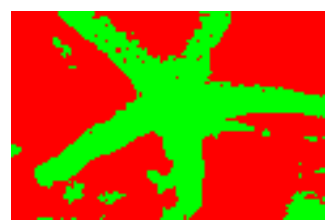

Figure 4.Output image of A2 
In third Algorithm (A3), the RGB image is taken as the input and it is converted into Luv color space. Figure 5 shows the Luv image. ( $u$ and $v$ ) chroma components of the Luv image are taken separately. SCCD is calculated for both the components. Remaining steps are same as the first Algorithm (A1). Figure 6 shows the output image of A3.

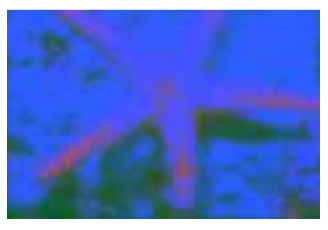

Figure 5.

LUV Image

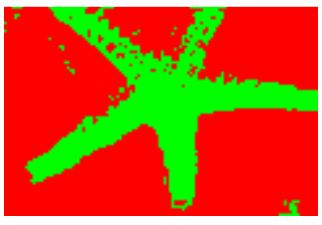

Figure 6.
Output image of A3

In the Fourth Algorithm (A4), the same procedure of (A3) is repeated with FCM instead of k-means clustering. Figure 7 shows the output image of A4.

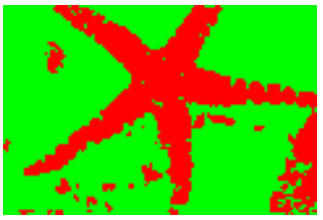

Figure 7.Output image of A4

Table 1: Performance Measure (Higher is better for RI, Lower is better for GCE, VOI and BDE)

\begin{tabular}{ccccc}
\hline Algorithms & $R I$ & $G C E$ & VOI & BDE \\
& & & & \\
\hline A1 & 0.587 & 0.365 & 2.432 & 2.999 \\
A2 & 0.640 & 0.192 & 1.910 & 2.348 \\
A3 & 0.649 & 0.159 & 1.824 & 2.053 \\
A4 & 0.690 & 0.125 & 1.643 & 1.799 \\
\hline
\end{tabular}

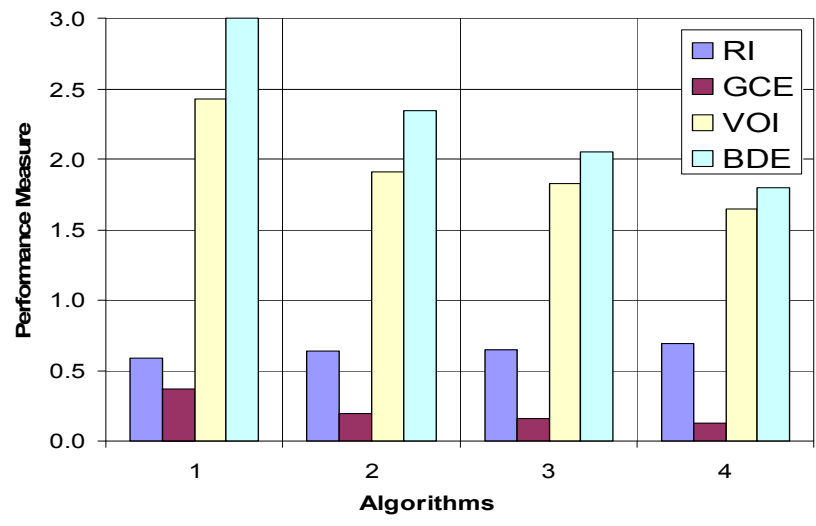

Figure 8. Performance of Algorithms A1,A2,A3 and A4.

\section{CONCLUSION}

Spectral clustering in Luv color space is performed by adopting spatial-color compactness function. Luv color space performs well when compared with the segmentation in RGB color space. The spectral clustering algorithm has k-means clustering, that is replaced by FCM and found better results than that of the latter one. Spectral clustering using FCM performed better segmentation in Luv color space compared to the algorithms A1, $\mathrm{A} 2$ and $\mathrm{A} 3$.

\section{REFERENCES}

[1] Busin L., Vandenbroucke N., Macaire L., and. Postaire J.G , 2005 "Color space selection for unsupervised colour image segmentation by analysis of connectedness properties". Robotics and Automation, vol. 20, no. 2, pp.70-77.Ding, W. and Marchionini, G. 1997 A Study on Video Browsing Strategies. Technical Report. University of Maryland at College Park.

[2] Chang H. and Yeung D., 2008 "Robust path-based spectral clustering". Pattern Recognition, vol. 41, no. 1, pp. 191203.Tavel, P. 2007 Modeling and Simulation Design. AK Peters Ltd.

[3] Chen T. Q. and Lu Y, 2002 ."Color image segmentation: an innovative approach". Pattern Recognition, vol. 35, no. 2, pp. 395-405.Forman, G. 2003. An extensive empirical study of feature selection metrics for text classification. J. Mach. Learn. Res. 3 (Mar. 2003), 1289-1305.

[4] Cheng H. D, Jiang X. H, Sun Y., and Wang J., 2001 “Color image segmentation : advances and projects" .Pattern Recognition, vol. 34, no. 12, pp. 2259-2281.

[5] Comaniciu and P. Meer ,2002 "Mean shift: a robust approach toward feature space analysis". Pattern Analysis and Machine Intelligence, vol. 24, no. 5, pp. 603-619.

[6] Deng and B. Manjunath, 2001 "Unsupervised segmentation of color-texture regions in images and video" .Pattern Analysis and Machine Intelligence, vol. 23, no. 8, pp. 800810.

[7] Fischer A. Y. N., Jordan M. I., and Weiss Y, 2002 "On spectral clustering: Analysis and an algorithm," Advances in Neural Information Processing Systems, vol. 14.

[8] Fischer B. and Buhmann J. M., 2003 "Bagging for pathbased clustering" . Pattern Anal. Mach. Intell., vol. 25, no. 11, pp. 1411-1415.

[9] Luxburg U. V, 2007 "A tutorial on spectral clustering," Statistics and Computing, vol. 17, no. 4, pp. 395-416.

[10] Macaire L, Vandenbroucke N, and Postaire J.G, 2006 "Color image segmentation by analysis of subset connectedness and color homogeneity properties," Computer Vision and Image Understanding, vol. 102, no. 1, pp. 105-116.

[11] Shi J. and Malik J , 2000 "Normalized cuts and image segmentation". Pattern Analysis and Machine Intelligence, vol. 22, no. 8, pp.888-905 
[12] Vandenbroucke N, Macaire L and Postaire J.G,2003 "Color image segmentation by pixel classification in an adapted hybrid color space. Application to soccer image analysis". Computer Vision and Image Understanding, vol. 90, no. 2, pp. 190-216.

[13] Veronica S. Moertini, 2002 "Introduction to five data clustering algorithms" INTEGRAL, Vol. 7, No. 2.

[14] Botte Lecocq C., Losson O. and Macaire L.,2007 "Color image segmentation by compacigram analysis," In Proceedings of the 14th International Conference on Image Analysis and Processing, Modena, Italy, pp. 212-215.

[15] Chang H. and Yeung D.Y, 2005 "Robust path-based spectral clustering with application to image segmentation", In Proceedings of the Tenth IEEE International Conference on Computer Vision, Washington, DC, USA, Volume 1, pp.278-285.

[16] Hebert P.A, Macaire L., 2008 "Spatial-Color pixel classification by spectral clustering for color image segmentation". In Proceedings of the 3rd IEEE International
Conference on Information and Communication Technologies: From Theory to Applications, Damascus (Syria).

[17] Martin D., Fowlkes C, Tal D, and Malik J , 2001 “A database of human segmented natural images and its application to evaluating segmentation algorithms and measuring ecological statistics". In Proceedings of the 8th International Conference on Computer Vision, vol. 2, Vancouver, BC, Canada, pp. 416-423.

[18] Vannoorenberghe P. and Flouzat G., 2006 “A belief-based pixel labeling strategy for medical and satellite image segmentation". In Proceedings of the IEEE Int. Conf. on Fuzzy Systems, Vancouver (Canada), pp. 1093-1098. 